Research Article

\title{
Hybrid Utilization of Loading Techniques and Cavity Groove for Performance Enhancement of the UWB (2-18GHz) Spiral Antenna
}

\author{
Muhammad Hamza $(D)$ and Wasif T. Khan \\ Electrical Engineering Dept., Lahore University of Management Sciences, Lahore 54792, Pakistan \\ Correspondence should be addressed to Muhammad Hamza; 17100141@lums.edu.pk
}

Received 25 June 2018; Accepted 31 July 2018; Published 23 August 2018

Academic Editor: Xiulong Bao

Copyright (C) 2018 Muhammad Hamza and Wasif T. Khan. This is an open access article distributed under the Creative Commons Attribution License, which permits unrestricted use, distribution, and reproduction in any medium, provided the original work is properly cited.

\begin{abstract}
This paper presents, for the first time, the hybrid use of loading techniques (which involve terminating the spiral arms with different structures to give a resistive or capacitive effect) and a novel technique of cavity groove in order to enhance the performance of twoarm, cavity-backed, $2-18 \mathrm{GHz}$ spiral antenna. The antenna is made on Duroid 5880 and has the smallest form factor $(50 \mathrm{~mm}$ diameter), meeting all the performance metrics, which are targeted for efficient performance of a spiral antenna for ultrawideband (UWB) applications. Measured results match the simulated results and show that by the utilization of hybrid loading and cavity groove, the gain is increased by a factor of $3.65 \mathrm{dBi}$ and the axial ratio is improved by $2.73 \mathrm{~dB}$ at $(2 \mathrm{GHz})$ as compared to a simple Archimedean antenna made on Duroid 5880, without increasing the size. The proposed antenna shows $2.25 \mathrm{~dB}$ more gain and improved axial ratio performance as compared to a simple Archimedean spiral antenna made on Tmm4, without the utilization of the hybrid techniques proposed earlier in the literature.
\end{abstract}

\section{Introduction}

Spiral antennas are circularly polarized (CP) antennas having very large bandwidth. Therefore, they are suitable to be used in applications such as wideband communication, navigation, and monitoring of the frequency spectrum [1]. Other applications include GPS, where it is advantageous to have RHCP antennas. For practical UWB $(2-18 \mathrm{GHz})$ systems, S11 matching below $-10 \mathrm{~dB}$ (i.e., VSWR $<2: 1$ ) and axial ratio less than $3 \mathrm{~dB}$ (for $\mathrm{CP}$ antennas) are normally targeted, both by commercial vendors and researchers. Moreover, for a $2-18 \mathrm{GHz}$ spiral antenna, the stated gain in literature across the whole band (especially lower frequencies) is mostly above $-5 \mathrm{dBi}$ (see Table 1). Considering these specifications, performance metrics listed in Table 2 are set as a benchmark for a decent, $2-18 \mathrm{GHz}$ spiral antenna.

Performance enhancement, keeping fixed antenna size, is an active research topic to improve the overall device/ antenna performance. To design smart and compact wireless products, we cannot rely on increasing the antenna size to achieve the desired results; therefore, researchers are actively looking for innovative solutions to improve the performance of spiral antennas while keeping the size constant. Some performance enhancement techniques include $3 \mathrm{D}$ rotation of spirals along cavity [2] and resistive loading [3], which are very hard to fabricate. Other easy-to-fabricate designs involve equiangular geometries [4] with tapering and meandering the spiral at its ends [5]. Careful utilization of these techniques can lead to either miniaturization or performance enhancement of the spiral antenna for a fixed antenna footprint. To the best of our knowledge, no one has presented in the literature the use of multiple loading techniques altogether in a " $2-18 \mathrm{GHz}$ " spiral antenna for performance enhancement.

In this paper, for the first time, we propose the smart utilization of hybrid loading techniques of meandering and tapering, as well as another novel technique of grooving the cavity, to boost the performance of a $2-18 \mathrm{GHz}$ spiral 
TABLE 1: Comparison of the proposed antenna with literature.

\begin{tabular}{|c|c|c|c|c|c|}
\hline Ref. & $\mathrm{BW}(\mathrm{GHz})$ & VSWR & Gain $(\mathrm{dBi})$ & Axial ratio (max. $\mathrm{dB})$ & Size $(\mathrm{mm})$ \\
\hline [8] & 2 to 18 & $<1.5: 1$ & -6 & 7 & 56 dia. \\
\hline [9] & 2 to 18 & - & -2 & 3.5 & $52 \times 52$ \\
\hline [10] & 2 to 10 & - & 0 & 2 & 72 dia. \\
\hline [11] & 2 to 18 & $<2: 1$ & -5 & 5 to 9 & $130 \times 130$ \\
\hline [12] & 2 to 18 & $3.9-12.6$ & 1.5 & $>5$ & $60 \times 60$ \\
\hline [13] (without hybrid techniques) & 2 to 18 & $<1.2: 1$ & -4.9 & 2.6 & 50 dia. \\
\hline This work & 2 to 18 & $<1.8: 1$ & $>-2.65$ & 2.27 & 50 dia. \\
\hline
\end{tabular}

TABle 2: Performance metrics for $(2-18 \mathrm{GHz})$ spiral antenna.

\begin{tabular}{lc}
\hline Performance metric & Benchmark value \\
\hline Boresight gain & At least $-5 \mathrm{dBi}$ \\
Boresight axial ratio & Below $3 \mathrm{~dB}$ \\
VSWR & Less than $2: 1$ \\
\hline
\end{tabular}

antenna within the smallest form factor (50 $\mathrm{mm}$ diameter). The $2-18 \mathrm{GHz}$ spiral antennas mentioned in the literature exceed the $50 \mathrm{~mm}$ size and do not meet the performance parameters listed in Table 2 simultaneously (one or another parameter is compromised). The comparison is provided in Table 1. Whereas, by the utilization of hybrid loading and cavity groove technique, we present the design of the smallest form factor with significant performance enhancement in gain by a factor of $3.65 \mathrm{dBi}$ and axial ratio improved by $2.27 \mathrm{~dB}$ as compared to a simple Archimedean spiral antenna, in an affordable, easy-to-fabricate design. In this paper, we also present the design guidelines for utilizing the aforementioned techniques and their detailed analysis with proper explanation and graphs. Not to skip, the role of the careful optimization of the design parameters, such as the microstrip width $(\mathrm{W})$, the number of turns, the spacing $(\mathrm{S})$ between the spiral arms, height of the cavity, and height of the substrate and accurate modelling of the absorber, is also explained. The paper is organized as follows:

Section 2 presents the detailed design flow, starting from the design guidelines and utilization of performance enhancement techniques, leading to modelling and fabrication of the spiral antenna. Section 2.1 draws the comparison between the measured and simulated results. Finally, Section 2.2 presents the conclusions and acknowledgments.

\section{Antenna Design}

The proposed antenna is two-arm, cavity-backed (metallic; for shielding) spiral antenna. The spirals start with simple Archimedean turns moving on to meandered (zigzag) spiral and finally tapering to a narrow microstrip width in the end. The antenna is fed through a tapered microstrip balun, having a low insertion loss. The balun serves as the impedance transformer, converting the single ended port to a symmetric port. Starting from unequal widths at the input to equal width of microstrips at the output, the balun transforms $50 \mathrm{ohms}$ unbalanced impedance to $130 \mathrm{ohms}$ balanced impedance which is a common input impedance

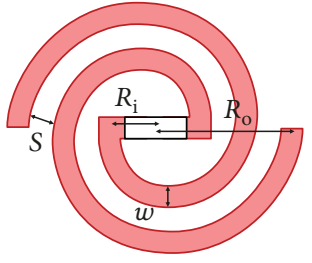

Figure 1: Simple Archimedean spiral antenna in the air.

among self-complimentary spiral antennas [6]. A novel technique of grooving the cavity is also added to enhance the performance.

2.1. Spiral Antenna Design Flow. A practical design of the spiral antenna requires that current attenuates as it propagates away from input terminals. After a certain point, the current is negligible and structure can be truncated at that point. This serves as the lower operating frequency cut-off point and is approximated to occur when wavelength is equal to circumference of spiral [7] as calculated by the following equation:

$$
f_{\text {low }}=\frac{c}{2 \pi R_{\text {outer }}}, \quad R_{\text {outer }}=24 \mathrm{~mm} \text { (in air). }
$$

Equation 1 suggests that in order to bring the lowest frequency of operation to $2 \mathrm{GHz}$, the minimum spiral diameter needs to be $48 \mathrm{~mm}$ in air (i.e., without substrate and not cavity-backed).

Reasonable matching is achieved throughout the band by keeping the microstrip width of the spiral arm $(W)$ and spacing between spiral arms $(S)$ almost equal, as shown in Figure 1. " $S$ " and " $W$ " are determined based upon the number of turns, given a fixed diameter. However, along with impedance matching, gain and axial ratio are also very critical parameters for a spiral antenna. For a $48 \mathrm{~mm}$ spiral in the air, the gain is very less at lower frequencies $(\sim-10 \mathrm{dBi}$ at $2 \mathrm{GHz}$ ) and axial ratio requirement is also not satisfied ( $\sim 6-$ $7 \mathrm{~dB}$ for most of the $2-18 \mathrm{GHz}$ band). Therefore, the ground plane is introduced to increase the gain. But the ground plane causes ripples/dips in the radiation pattern at higher frequencies and deteriorates the axial ratio at frequencies for which the spacing between the ground plane and spiral (" $A_{\mathrm{t}}+$ air gap," see Figure 2) is not quarter-wavelength. If we try to achieve better matching at higher frequencies by adjusting the ground plane height, the lower frequencies get mismatched and vice versa. 


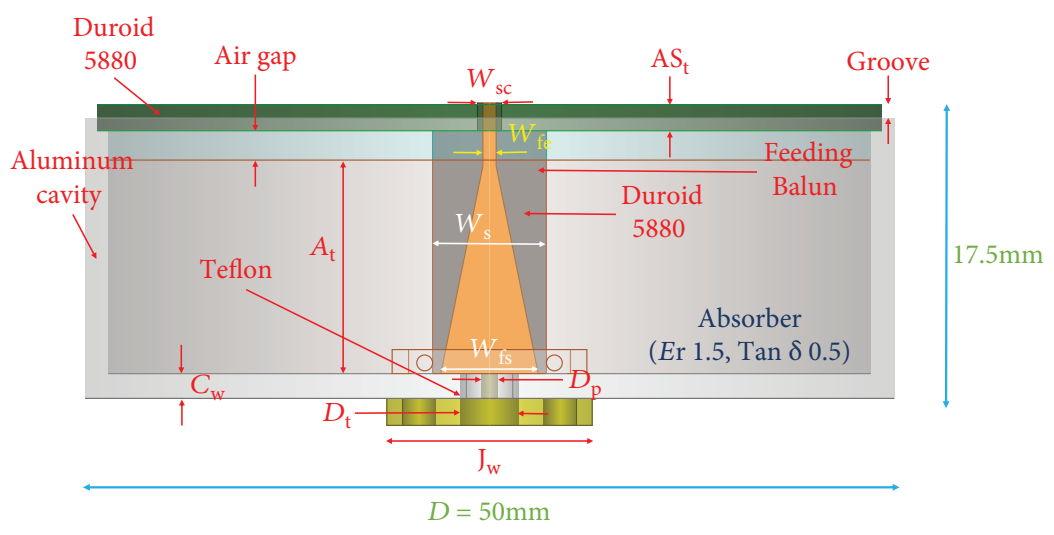

Figure 2: Side view of the proposed spiral antenna.

TABLE 3: Dimensions of spiral antenna.

\begin{tabular}{lccccccc}
\hline Parameter & Value $(\mathrm{mm})$ & Parameter & Value $(\mathrm{mm})$ & Parameter & Value $(\mathrm{mm})$ & Parameter & Value $(\mathrm{mm})$ \\
\hline$D$ & 50 & $\mathrm{AS}_{\mathrm{t}}$ & 1.575 & $S$ & 0.8 & $W_{\mathrm{s}}$ & 7 \\
$D_{\mathrm{p}}$ & 1 & $A_{\mathrm{t}}$ & 13.2 & us $\_\mathrm{w}$ & 0.7 & $W_{\mathrm{fs}}$ & 6 \\
$D_{\mathrm{t}}$ & 3.6 & $n 1_{\text {simple turns }}$ & 6.5 & $C_{\mathrm{w}}$ & 1.5 & $W_{\mathrm{fe}}$ & 0.8 \\
$R_{\mathrm{o}}$ & 22.8 & $n 2_{\text {meandering }}$ & 0.5 & Air gap & 1.8 & $W_{\mathrm{sc}}$ & 1.5 \\
$R_{\mathrm{i}}$ & 0.92 & $n 3_{\text {tapering }}$ & 0.5 & Groove & 0.787 & $J_{\mathrm{w}}$ & 12.7 \\
\hline
\end{tabular}

To solve the matching issue, substrate is used, which reduces the wavelength due to its relative permittivity (i.e., $\lambda_{\mathrm{r}}=\lambda_{\mathrm{o}} / \varepsilon_{\mathrm{r}}$ ), and smaller frequencies get matched at the same ground plane spacing. The substrate also gives support to the spiral antenna. To improve the axial ratio, an absorber is introduced between the antenna and ground plane which absorbs waves going towards and coming back (if any) from the ground plane that cause destructive interference. Optimal absorber thickness $(\sim 13 \mathrm{~mm}$ in our case) is chosen based on the loss tangent of absorber and frequencies of interest (which need to be absorbed). The thicker the absorber is, the lesser will be the gain and the better will be the axial ratio. In our case, we chose a honeycomb absorber (HC-55175, ARC Tech.) and modelled it in HFSS as a solid material with $E_{\mathrm{r}} 1.5$ and $\tan \delta 0.5$ [8].

Another problem that needs to be addressed is the currents that get reflected from open ends of spiral which radiate and interfere with main radiation beam degrading the axial ratio. Increasing number of turns solves this problem as the reflected currents get attenuated before reaching the main radiation region of the spiral, which is near to the centre. But, it decreases the gain because for a fixed antenna size; the microstrip width needs to be decreased in order to increase the turns, which increases the $I^{2} R$ losses and reduces the overall gain. Increasing the substrate permittivity $\left(\varepsilon_{\mathrm{r}}\right)$ decreases the relative wavelength $\left(\lambda_{\mathrm{r}}\right)$ at $2 \mathrm{GHz}$ (i.e., $\lambda_{\mathrm{r}}=\lambda_{\mathrm{o}}$ $\left./ \varepsilon_{\mathrm{r}}\right)$, and therefore, smaller size is required by an antenna to radiate at $2 \mathrm{GHz}$. This increases the electrical length of the antenna and enhances the performance at $2 \mathrm{GHz}$. Optimum permittivity of substrate is between 2 and 5 for the optimum electrical length of the antenna, but very large permittivity will increase surface waves and deteriorate the axial ratio.
Therefore, for substrates with higher $E_{\mathrm{r}}(>4)$, the substrate thickness should be kept small $(0.5-1 \mathrm{~mm})$ to improve the axial ratio.

Shape of the spiral, number of turns, substrate thickness, electrical properties, size of absorber, and ground plane spacing play a critical role in meeting the requirements of the gain and axial ratio. By optimizing all the aforementioned parameters on a $50 \mathrm{~mm}$ dia., simple Archimedean spiral antenna, the gain and axial ratio at $2 \mathrm{GHz}$ were still $-6 \mathrm{dBi}(<-5 \mathrm{dBi})$ and $5 \mathrm{~dB}(>3 \mathrm{~dB})$ while the VSWR was $<2: 1$ throughout the band. Increasing the spiral diameter increases the physical length of the antenna and improves both the gain and axial ratio. Therefore, people usually increase the spiral diameter from $48 \mathrm{~mm}$ to at least $55 \mathrm{~mm}$ (even $\sim 70 \mathrm{~mm}$ dia. is mentioned in Table 1), in order to achieve reasonable performance in all aspects. But for a miniaturized UWB systems, this $(7 \mathrm{~mm})$ increase in diameter becomes a big deal, necessitating to find out innovative solutions to achieve the required results without compromising the antenna size. Therefore, along with optimization of parameters, we propose some performance enhancement techniques that can be utilized for achieving the required benchmarks without increasing the size. The final optimized parameters are tabulated in Table 3.

2.2. Performance Enhancement Techniques. Our proposed design involves the smart use of three techniques simultaneously to achieve the required results:
(a) Tapering
(b) Meandering 


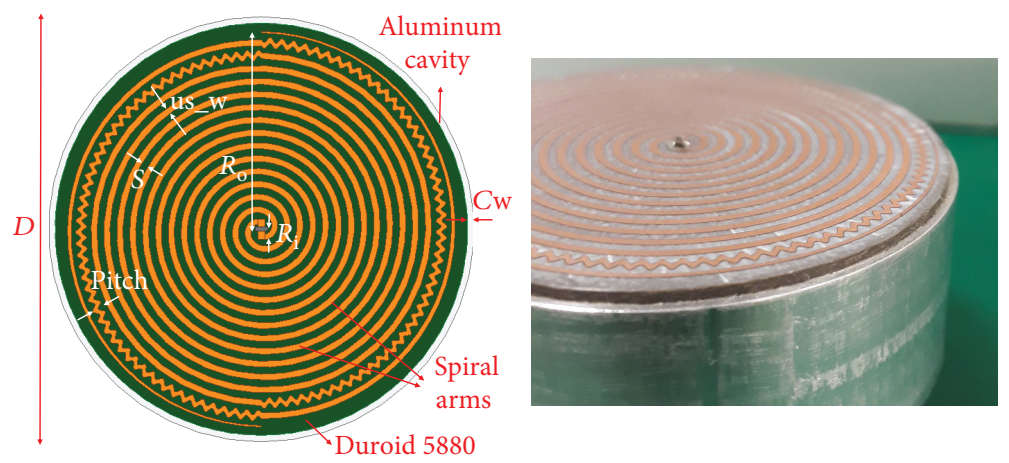

Figure 3: Top view and close-up of the cavity groove.

(c) Groove in the cavity (novel)

The loading techniques of meandering and tapering are very briefly mentioned in the literature (used individual/separately and not as a combination of two techniques) while the technique of making a "groove in the cavity" is completely novel. Usually, the higher frequencies already show satisfactory results without the use of these techniques, but the low-frequency gain and axial ratio of the spiral antenna remain unsatisfactory.

2.2.1. General Trends and Effects. In this section, the effect and general trends of each of the loading techniques will be discussed one by one and then their combined effect will be discussed in the next section:

Starting off with meandering, meandering (zigzag) of spiral arms increases the antenna length by introducing zigzag turns in the spiral arms and reducing the wave velocity at the low-frequency portion of the spiral. This decreases the low-frequency operational point of the antenna [5]. Since the lowest operating frequency of the spiral antenna is approximated to occur when the wavelength equals the length of the spiral arm [7], therefore, by making the zigzag turns in the spiral arms, the length of the spiral arms increases which increases the physical length of the antenna and consequently, the impedance matching at lower frequencies is achieved. The pitch refers to the amount of the zigzag that is made in the spiral arm (see Figure 3, top view); the more the pitch, the more frequently the spiral is meandered.

Figure 4 shows that the gain continues to improve as we increase the pitch (zigzag) of the meandering but the axial ratio increases and then fluctuates between 5.7 and $6 \mathrm{~dB}$ as we increase the pitch after a certain value (0.6). This is because after a certain pitch, the zigzag arms come in close proximity to the adjacent arms and cause interference; this is one of the reasons that spiral arm was not meandered more than half a turn. We chose a pitch of 0.9 to ensure higher gain, compromising the axial ratio, which can be improved by tapering.

The second technique, tapering (decreasing the microstrip width of the spiral arms as they extend to the far end) is very useful in order to improve the axial ratio of the antenna. One of the main reasons of the bad axial ratio is the currents that reflect back from the open ends of the spiral and radiate destructively; tapering the ends of the spiral

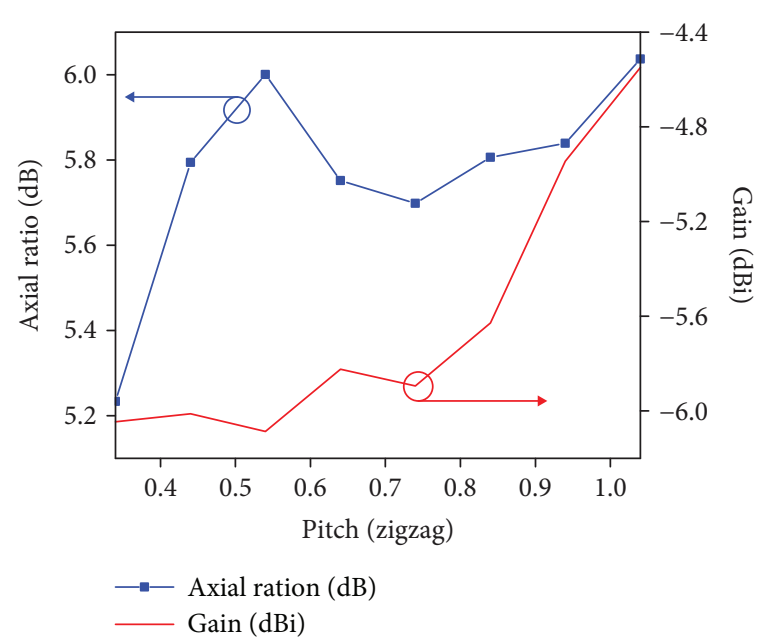

Figure 4: Trend of gain and axial ratio with meandering.

means narrowing the microstrip width which increases the resistance and results into higher $I^{2} R$ losses. Due to this, most of the current attenuates as it moves towards the end of the spiral and very less of it reflects back, improving the axial ratio.

Tapering is a very good technique because instead of decreasing the overall width of the spiral arm, which will decrease the gain significantly, we just taper it in the end to improve the axial ratio of the whole band while keeping the gain roughly the same. The effect of tapering is shown in Figure 5(a); the axial ratio tends to improve as we increase the tapering of the microstrip while the gain slightly decreases. We tapered the spiral up till $0.6 \mathrm{~mm}$ to ensure maximum improvement in the axial ratio.

Lastly, a novel technique to improve the performance is by introducing a groove in the cavity as shown in Figure 2. The groove is defined as a reduction in the metallic sidewall adjacent to the substrate in order to prevent the reflections of the surface waves in the substrate from the metallic cavity and decrease the unwanted self-interference of the fields. As we increase depth of the groove, the reflections decrease and the gain and axial ratio improve, as shown in Figure 5(b). Cavity groove is quite an easy and efficient way to improve the performance of a spiral antenna. However, the groove was kept to half of the thickness of the substrate (i.e., 


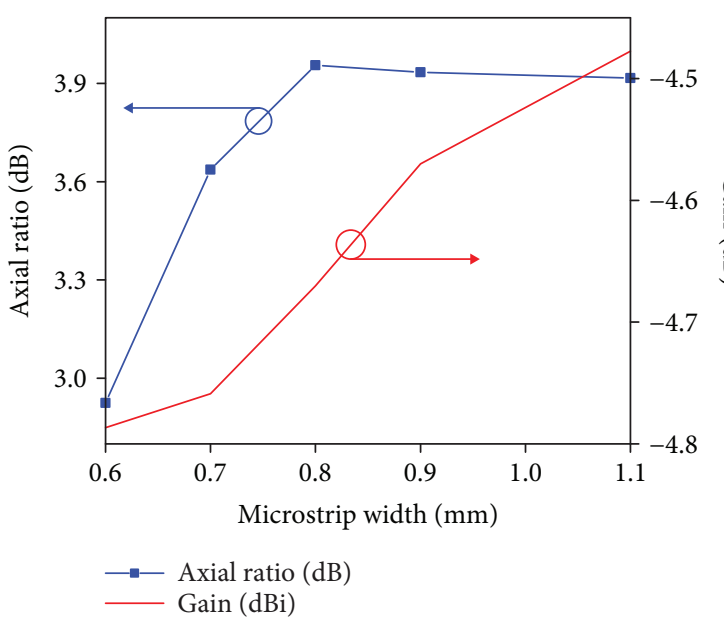

(a)

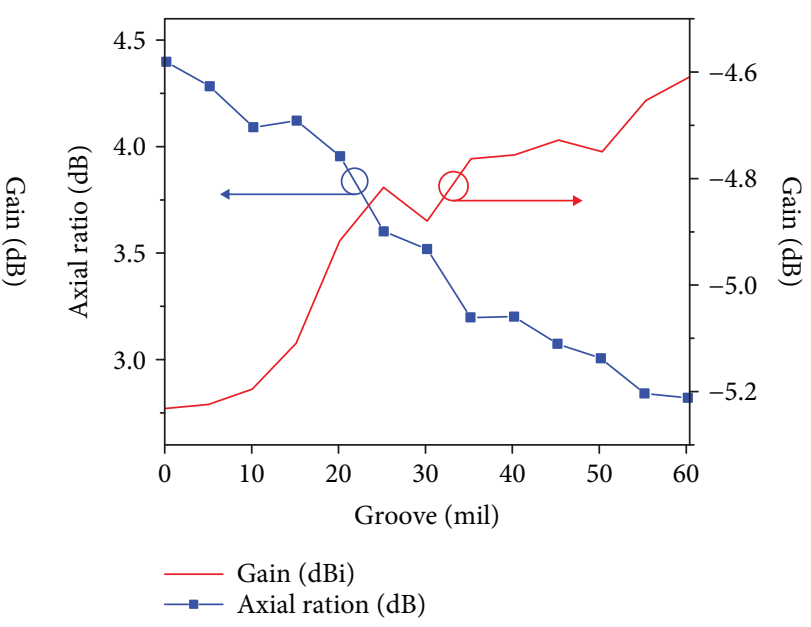

(b)

FIgURE 5: Trend of gain and axial ratio with (a) tapering and (b) cavity groove.

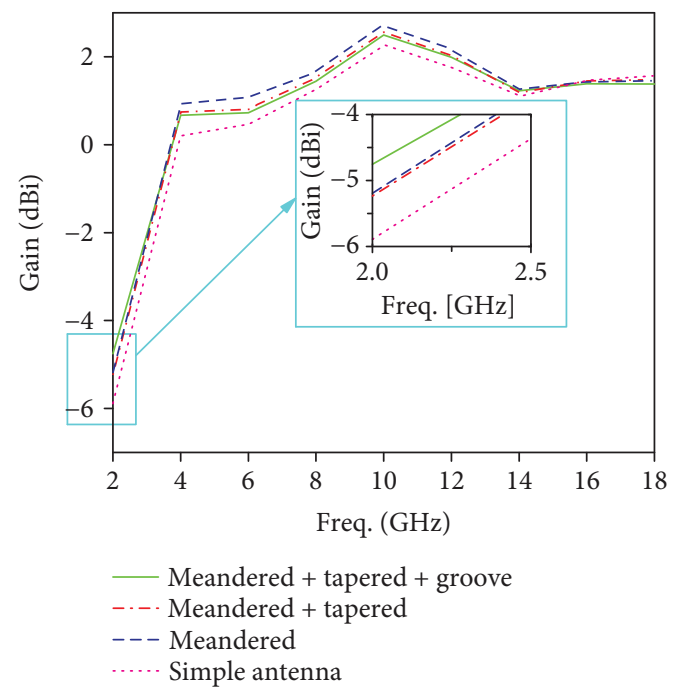

(a)

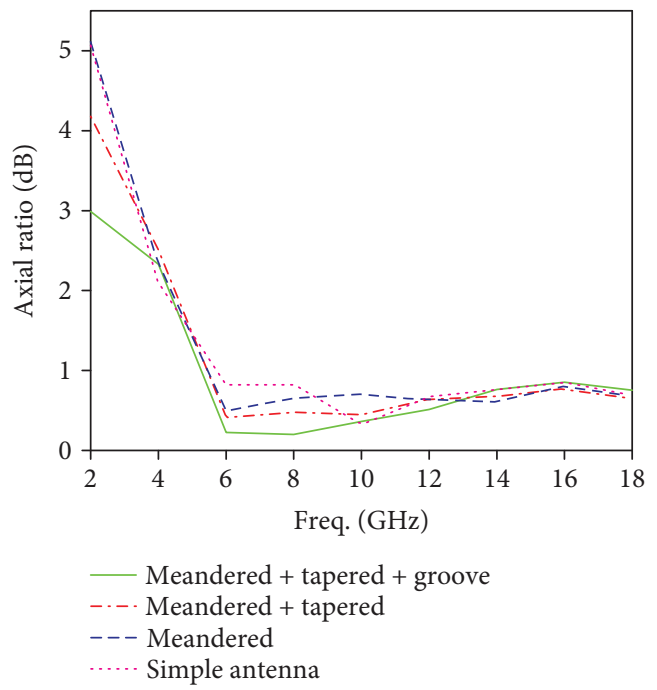

(b)

FiguRE 6: Effect of meandering, tapering, and groove on (a) gain and (b) axial ratio.

$\sim 31 \mathrm{mil}=0.787 \mathrm{~mm}$ ) in order to provide support to the spiral. While, in the case of radome, the groove can be further increased and even better results can be achieved because radome can provide the support. This makes the respective technique very powerful and advantageous.

2.2.2. Combination of Techniques. As discussed in the previous section, meandering improved the gain from -6 to $-5.1 \mathrm{dBi}$ at $2 \mathrm{GHz}$, as shown in Figure 5(b) but the axial ratio was still around $5 \mathrm{~dB}$ which was improved by proper termination of the spiral arms, by tapering. Hence, meandering alone was not sufficient to get improved results in all aspects.

Therefore, tapering was also implemented to terminate the spiral. This effect is also visible in Figure 6; the axial ratio improved from $5.1 \mathrm{~dB}$ to $\sim 4.15 \mathrm{~dB}$ at $2 \mathrm{GHz}$ while the gain decreased negligibly. Unlike other techniques, the increase in depth of the groove significantly improved the gain from $-5.1 \mathrm{dBi}$ to $-4.7 \mathrm{dBi}$ and axial ratio from $4.15 \mathrm{~dB}$ to $2.9 \mathrm{~dB}$. Using cavity groove and the other two mentioned techniques altogether, we were able to improve the performance of the spiral antenna throughout the band, while maintaining the smallest form factor, with gain increased by $3.65 \mathrm{dBi}$ and axial ratio improved by $2.27 \mathrm{~dB}$ at $2 \mathrm{GHz}$ as compared to a simple Archimedean spiral antenna that does not utilize these techniques.

2.3. Antenna Model. A 3D model of the antenna is shown in Figure 7. The detailed dimensions of design have been tabulated in Table 3 as well as marked in the pictures to match the dimensions precisely.

2.4. Antenna Fabrication. The antenna cavity was made using a lathe machine. The substrate and the balun PCB were 

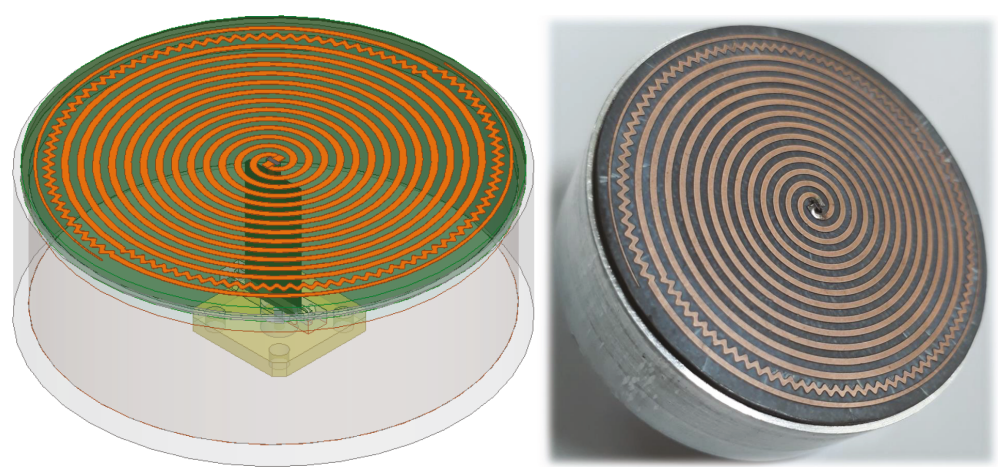

FIGURE 7: 3D model and fabricated proposed spiral antenna.

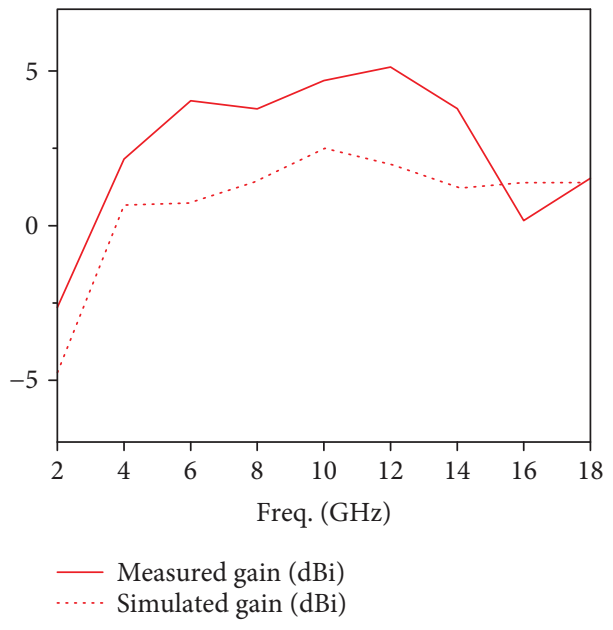

(a)

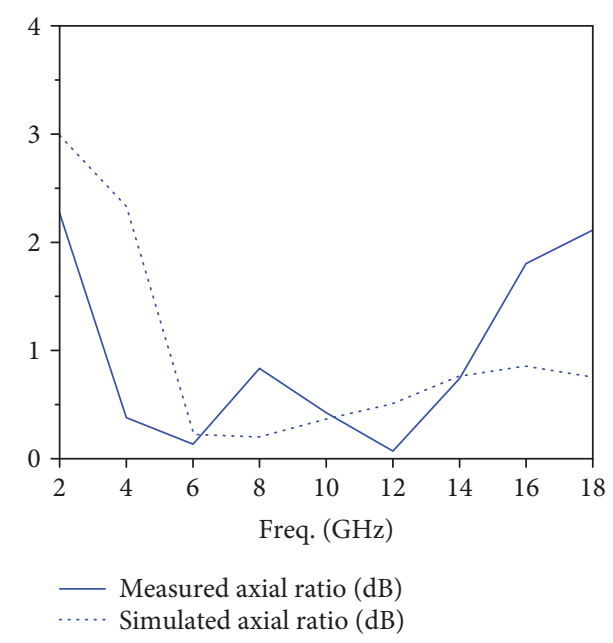

(b)

FIgURE 8: Comparison of measured and simulated (a) gain and (b) axial ratio.

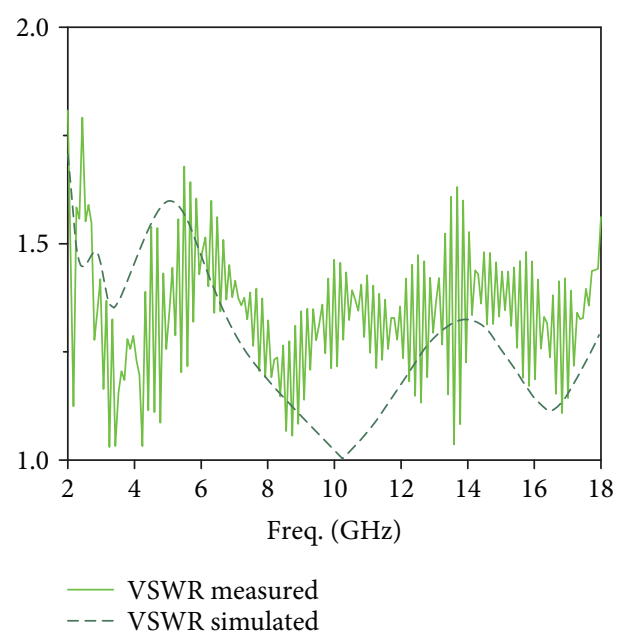

FIGURE 9: Comparison of measured and simulated VSWR.

fabricated using the LPKF PCB rapid prototyping machine. Honeycomb absorber was used and modelled as a solid material $\left(E_{\mathrm{r}} 1.5\right.$, loss tangent 0.5$)$ [8] in HFSS. The balun was passed through the hole made in the honeycomb absorber till the cavity base. The metallic cavity was grounded with the connector. The differential signal from both ends of the balun was fed to each spiral arm by soldering both microstrip sides of the balun with the spiral arms.

\section{Measurements and Results}

The proposed spiral antenna has been simulated in an Ansoft HFSS-v16 EM simulator. Figures 8 and 9 show the comparison of measured and simulated results (gain, axial ratio, and VSWR) of the spiral antenna along with the frequency.

Figure 10 shows the measured and simulated radiation pattern of the spiral antenna. The measured results bear slightly improved offset, in the gain and axial ratio as shown by the graphs in Figure 10. The reason can be the extra filing of the groove in the cavity (i.e., greater than $31 \mathrm{mil}=0.787 \mathrm{~mm}$ ) as compared to the simulation model and in turn improving the results. The results serve as a proof of the success of our design. More precisely, the axial ratio is far less than $3 \mathrm{~dB}$ (i.e., $<2.27 \mathrm{~dB}$ ) throughout the $2-18 \mathrm{GHz}$ band, gain is greater than $-2.65 \mathrm{~dB}$, while the requirement was just $>-5 \mathrm{~dB}$, and similarly, VSWR is less than 1.70 all over the $2-18 \mathrm{GHz}$ bandwidth. 


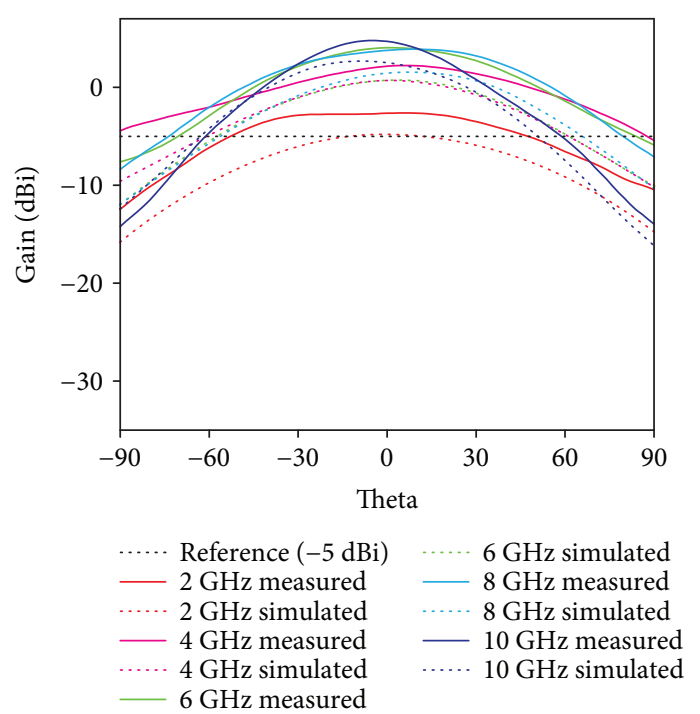

(a)

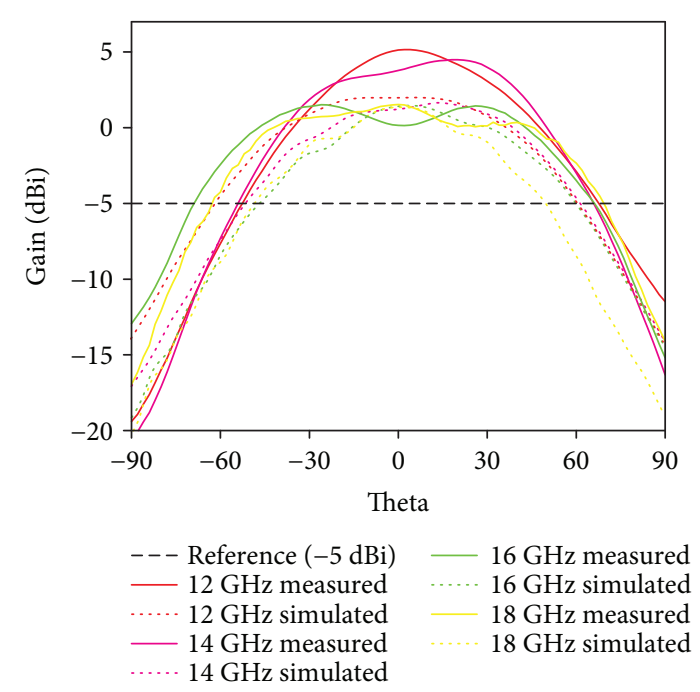

(b)

Figure 10: Comparison of measured (solid line) and simulated (dotted line) radiation pattern from (a) 2-10 GHz and (b) $11-18 \mathrm{GHz}$.

As can be seen from Table 1, by the utilization of hybrid loading and cavity groove technique, the antenna has performed significantly better than the already mentioned antennas in the literature. Moreover, the proposed antenna shows $2.25 \mathrm{~dB}$ more gain and improved axial ratio performance as compared to a simple Archimedean spiral antenna made on Tmm4, without the utilization of the hybrid techniques [13].

\section{Conclusion}

The work presented in this paper demonstrates for the first time the smart utilization of loading techniques along with a new technique of cavity groove to develop a $2-18 \mathrm{GHz}$, compact (50 $\mathrm{mm}$ diameter), cavity-backed, spiral antenna with significantly enhanced performance in all aspects than the spiral antennas mentioned in literature. The effect of hybrid loading and cavity groove technique to improve the performance of an ultra-wideband $(2-18 \mathrm{GHz})$ spiral antenna is presented with detailed graphs and trends. Measured results show significant improvement in the gain and axial ratio throughout the band, with gain increased by a factor of $3.65 \mathrm{dBi}$ and axial ratio by $2.7 \mathrm{~dB}$ at $2 \mathrm{GHz}$. The proposed antenna is suitable to fit in miniaturized products and has applications in wideband sensing, spectrum monitoring, radar systems, and broadband communication.

\section{Data Availability}

The data used to support this study is taken from online research articles available in IEEE Xplore and Wiley Online Library.

\section{Conflicts of Interest}

The authors declare that there is no conflict of interest regarding the publication of this paper.

\section{Acknowledgments}

The authors would like to thank RWR Pvt Ltd. for their fabrication and measurement facility for spiral antenna. This work has been funded by RWR Pvt Ltd.

\section{References}

[1] S. E. Lipsky, Microwave Passive Direction Finding, SciTech Publishing, 2004.

[2] Y. W. Zhong, G. M. Yang, J. Y. Mo, and L. R. Zheng, "Compact circularly polarized Archimedean spiral antenna for ultrawideband communication applications," IEEE Antennas and Wireless Propagation Letters, vol. 16, pp. 129-132, 2017.

[3] M. Zou, J. Pan, P. Li, and L. Zuo, "Design and performance of a lumped resistively loaded spiral antenna," in Proceedings of the 2012 IEEE International Symposium on Antennas and Propagation, pp. 1-2, Chicago, IL, USA, November 2012.

[4] R. Chernobrovkin, I. Ivanchenko, V. Pischikov, and N. Popenko, "UWB equiangular spiral antenna for 7.5$40 \mathrm{GHz}$," Microwave and Optical Technology Letters, vol. 54, no. 9, pp. 2190-2194, 2012.

[5] J. M. O'Brien, J. E. Grandfield, G. Mumcu, and T. M. Weller, "Miniaturization of a spiral antenna using periodic Z-plane meandering," IEEE Transactions on Antennas and Propagation, vol. 63, no. 4, pp. 1843-1848, 2015.

[6] S. A. P. Rizvi and R. A. A. Khan, "Klopfenstein tapered 2$18 \mathrm{GHz}$ microstrip balun," in Proceedings of 2012 9th International Bhurban Conference on Applied Sciences \& Technology (IBCAST), pp. 359-362, Islamabad, Pakistan, January 2012.

[7] M. Asad, J. Gilani, A. Khalid, and M. S. Iqbal, "Optimizing Q factor of square spiral antenna," in Second Pacific-Asia Conference on Circuits, Communications and System, Beijing, China, August 2010.

[8] C. M. Seong and D. C. Park, "Design of cavity-backed spiral antennas," in Proceedings of 2012 5th Global Symposium on Millimeter-Waves, pp. 186-190, Harbin, China, May 2012. 
[9] N. Rahman and M. N. Afsar, "A novel modified Archimedean polygonal spiral antenna," IEEE Transactions on Antennas and Propagation, vol. 61, no. 1, pp. 54-61, 2013.

[10] H. Oyanagi, H. Nakano, J. Yamauchi, and S. Sasaki, "Cavitybacked Archimedean spiral antenna with strip absorber," IET Microwaves, Antennas \& Propagation, vol. 2, no. 7, pp. 725730, 2008.

[11] W. Fu, E. R. Lopez, W. S. T. Rowe, and K. Ghorbani, “A planar dual-arm equiangular spiral antenna," IEEE Transactions on Antennas and Propagation, vol. 58, no. 5, pp. 1775-1779, 2010.

[12] T. W. Eubanks and K. Chang, "A compact parallel-plane perpendicular current feed for a modified equiangular spiral antenna," IEEE Transactions on Antennas and Propagation, vol. 58, no. 7, pp. 2193-2202, 2010.

[13] A. Ali, M. Hamza, and W. T. Khan, "Smallest form factor, high performance $2-18 \mathrm{GHz}$ cavity-backed Archimedean spiral antenna," in 2017 International Symposium on Antennas and Propagation (ISAP), pp. 1-2, Phuket, Thailand, November 2017. 


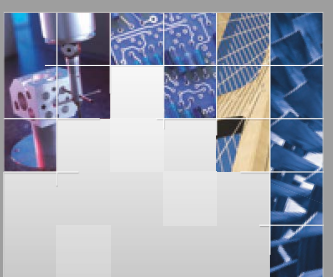

\section{Enfincering}
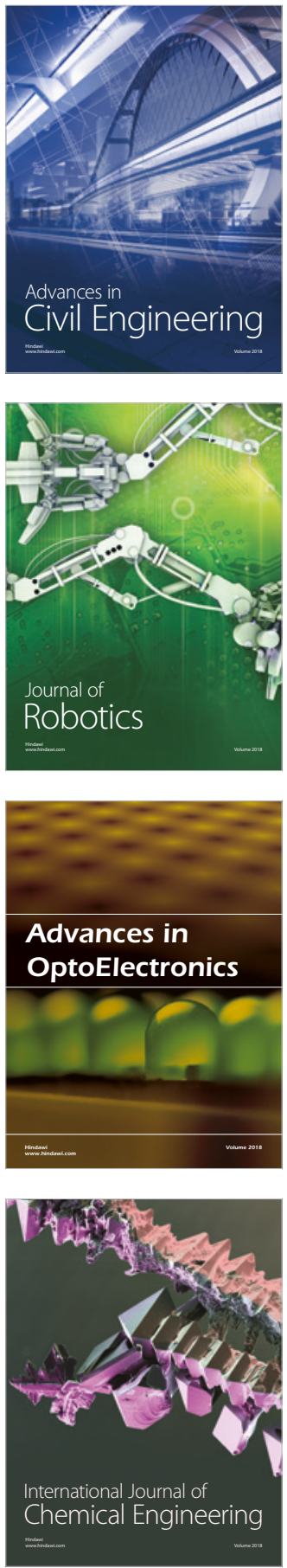

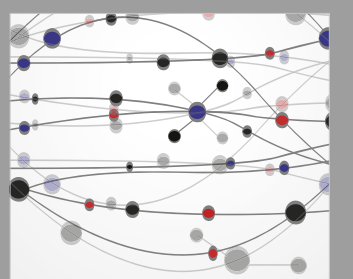

\section{Rotating \\ Machinery}

The Scientific World Journal

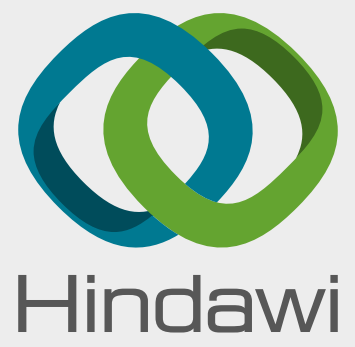

Submit your manuscripts at

www.hindawi.com
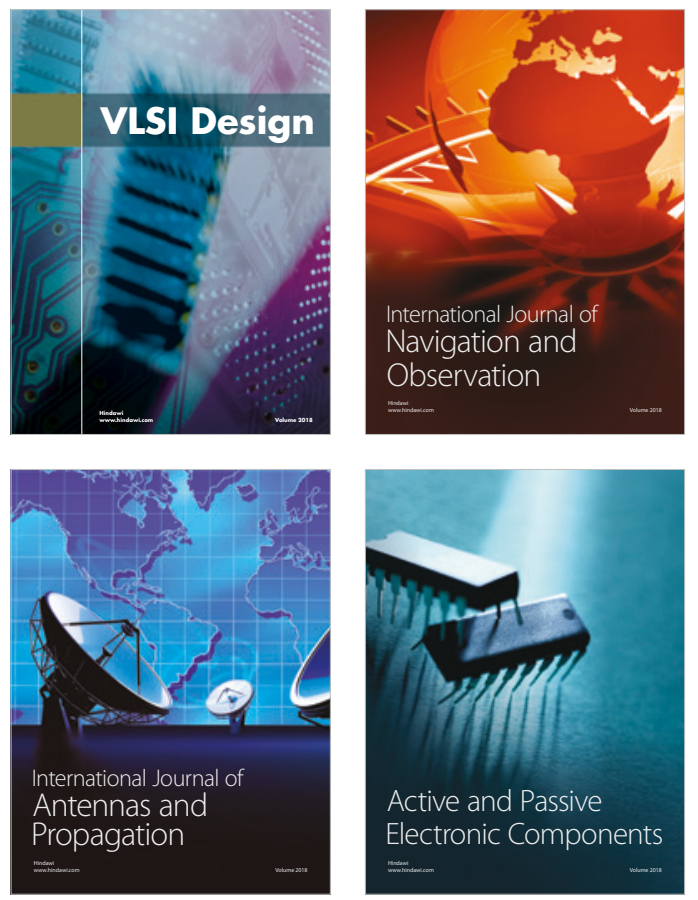
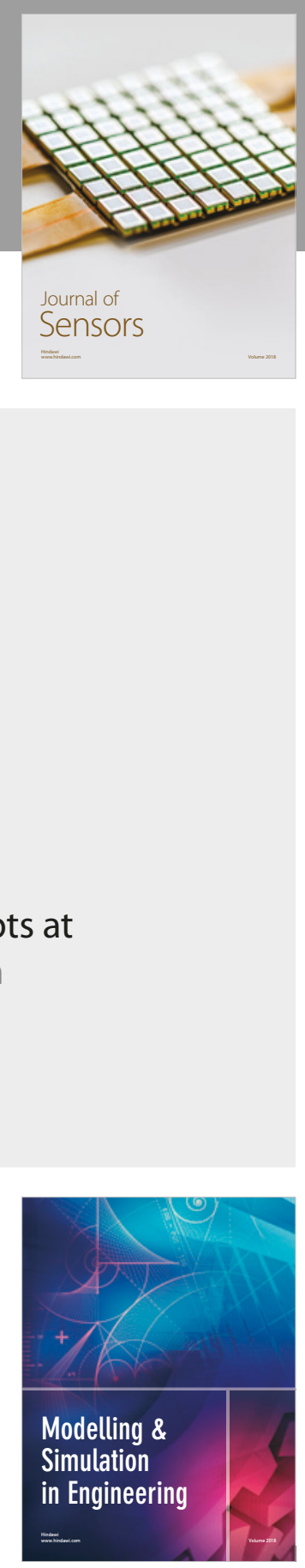

\section{Advances \\ Multimedia}
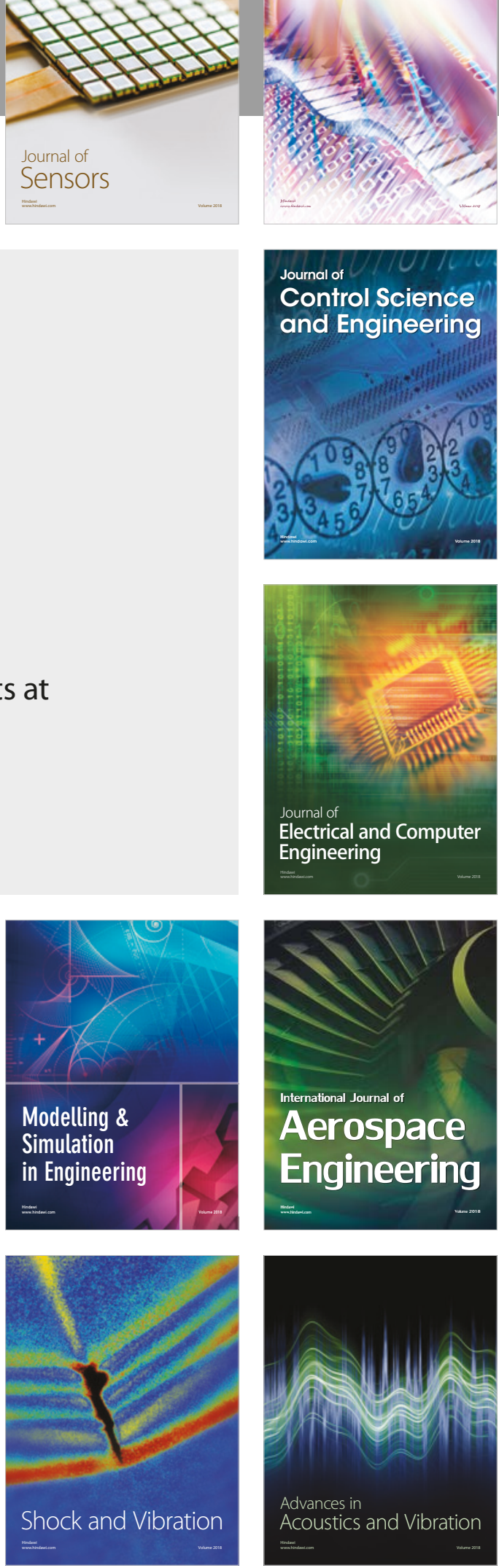